\title{
Ligny-le-Châtel (Yonne) : étude typologique et pétrographique des sarcophages
}

\section{Fabrice Henrion et Stéphane Büttner}

\section{Q OpenEdition \\ 1 Journals}

Édition électronique

URL : https://journals.openedition.org/cem/180

DOI : $10.4000 /$ cem. 180

ISSN : 1954-3093

\section{Éditeur}

Centre d'études médiévales Saint-Germain d'Auxerre

\section{Édition imprimée}

Date de publication : 15 août 2006

ISSN : 1623-5770

\section{Référence électronique}

Fabrice Henrion et Stéphane Büttner, «Ligny-le-Châtel (Yonne) : étude typologique et pétrographique des sarcophages », Bulletin du centre d'études médiévales d'Auxerre / BUCEMA [En ligne], 10 | 2006, mis en ligne le 13 octobre 2006, consulté le 22 septembre 2022. URL : http://journals.openedition.org/ cem/180 ; DOI : https://doi.org/10.4000/cem.180

Ce document a été généré automatiquement le 22 septembre 2022.

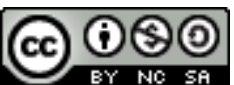

Creative Commons - Attribution - Pas d'Utilisation Commerciale - Partage dans les Mêmes Conditions 4.0 International - CC BY-NC-SA 4.0

https://creativecommons.org/licenses/by-nc-sa/4.0/ 


\title{
Ligny-le-Châtel (Yonne) : étude typologique et pétrographique des sarcophages
}

\author{
Fabrice Henrion et Stéphane Büttner
}

1 A la demande conjointe du Service régional de l'archéologie de Bourgogne et de l'INRAP Grand-Est Sud, nous avons eu en charge l'étude typologique et pétrographique des sarcophages de pierre de la nécropole de «La Coupe au Meunier » à Ligny-le-Châtel, fouillée de mai à novembre 2005 sous la direction de Patrick Chopelain (INRAP). Le site a livré 64 sarcophages, ce qui représente $38 \%$ des inhumations inventoriées et fouillées ${ }^{1}$. À cela, il conviendrait d'ajouter pour mémoire les exemplaires rencontrés à l'occasion du diagnostic mené en 2001, ainsi que la «cinquantaine de sarcophages découverts entre 1760 et 1825 » signalée dans l'Histoire de la ville de Ligny-le-Châtel de l'abbé Cornat ${ }^{2}$.

2 Une des principales caractéristiques de ces sarcophages est leur relative homogénéité typologique et lithologique. Avec une forte prédominance du Groupe D (taille et finitions à la polka) et des Types lithologiques 2 et 3 (Kimméridgiens), l'approvisionnement apparaît comme très local, sans que l'on puisse toutefois parler d'exclusivité de ces centres de production puisque d'autres faciès sont utilisés de façon concomitante. Par contre, il apparaît que les carrières situées dans les niveaux kimméridgiens (Calcaire de Tonnerre) se spécialisent dans la production du Groupe D. Bien que celles situées dans la zone du récif oxfordien de l'Yonne en produisent également (par exemple le S. 037), leur diffusion semble plus modeste ici.

3 Une plus grande diversité lithologique apparaît pour les sarcophages du Groupe B (taille et finition au pic ou à la broche), alors que ceux du Groupe C (dit par ailleurs «bourguignon-champenois ») proviennent exclusivement des calcaires oxfordiens de la zone d'avant-récif. Ce constat alimente la difficile question de savoir si le choix d'un modèle de sarcophage est imposé par sa disponibilité ou au contraire laissé à l'appréciation individuelle. Dans le cas de Ligny, on peut penser que l'approvisionnement se fait habituellement (ou traditionnellement) depuis les carrières 
de la vallée du Serein (proximité et facilité de transport), mais on assiste ponctuellement à des importations depuis les vallées de l'Yonne et de la Cure, sans que l'on puisse pour l'instant en saisir les motivations.

4 Le schéma proposé pour Ligny s'accorde tout à fait avec ce qui a pu être reconnu sur d'autres sites, comme à Escolives-Sainte-Camille (rive gauche de l'Yonne) par exemple où les carrières de la région de Bailly/Vincelottes (rive droite de l'Yonne) sont largement sollicitées au détriment de celles situées dans la zone du récif. Un autre cas, celui de Quarré-les-Tombes (Yonne), montre des productions presque exclusivement issues des calcaires récifaux (Type $4 \mathrm{~b}$ - zone d'avant-récif, Arcy-sur-Cure/ SaintMoré ?) avec une probable diffusion par la Cure et une rupture de charge à Sermizelles, voire même à Saint-Père, puis un transport terrestre sur moins de vingt kilomètres ${ }^{3}$.

5 La confrontation de la typologie avec la lithologie apporte également un éclairage particulier à l'étude de la gestion des carrières. A titre d'exemple, les S. 007 et S. 074, tous deux du Groupe D, présentent chacun pour la cuve et le couvercle deux faciès différents (Type 2 et Type 3) mais appartenant au même étage. Cuve et couvercle sont probablement issus de la même carrière, mais pas du même front ou du même niveau (choix en fonction de la qualité du matériaux, de l'épaisseur du banc, etc.), ni peut-être des mêmes carriers; d'où l'indispensable modélisation et standardisation de la production. En outre, ces indices impliquent que la carrière est à ciel ouvert, car en souterrain la liberté d'exploitation est plus limitée.

6 Le S. 049 se distingue des autres sarcophages du Groupe D par son décor ${ }^{4}$, mais également par sa lithologie. Le couvercle est taillé dans un calcaire du Type lithologique 2 (Kimméridgien), alors que la cuve renvoie au Type lithologique 5 (Oxfordien?), deux faciès bien distincts géographiquement. Deux solutions peuvent être proposées: soit il s'agit d'un remploi, soit ce sarcophage procède d'un mode opératoire atypique. La première solution nous semble difficilement envisageable dans la mesure où d'une part, les dimensions du couvercle semblent correspondre à celles de la cuve, et d'autre part, à la rareté du décor de la cuve s'ajoute celle de la typologie du couvercle (de section rectangulaire, angles arrondis, évidé) et de son traitement technique (polka utilisée en finition). On imagine mal qu'autant de particularismes se trouvent réunis par le fruit du hasard. Quant à la seconde solution, bien qu'elle ne réponde pas aux schémas reconnus jusqu'ici, elle pourrait illustrer le cas d'une production de sarcophage en dehors du lieu d'extraction, dans un atelier se fournissant auprès de plusieurs carrières, et selon un modèle propre. Nous avons conscience de la fragilité de cette hypothèse mais elle a au moins le mérite d'ouvrir un nouveau champ de réflexion.

7 D'un point de vue typochronologique, l'utilisation de sarcophages dans la nécropole de Ligny semble bien se placer à partir de l'extrême fin du $\mathrm{VI}^{\mathrm{e}}$ siècle et couvrir tout le siècle suivant. Au-delà d'une stricte étude des objets, il serait indispensable d'intégrer les données d'ores et déjà acquises aux résultats de l'étude globale du site, actuellement en cours. Non seulement pour en préciser la chronologie, mais surtout pour mieux comprendre les relations entre les inhumations en sarcophage et les autres, les regroupements d'objets typologiquement différents ou encore l'économie d'une nécropole. 


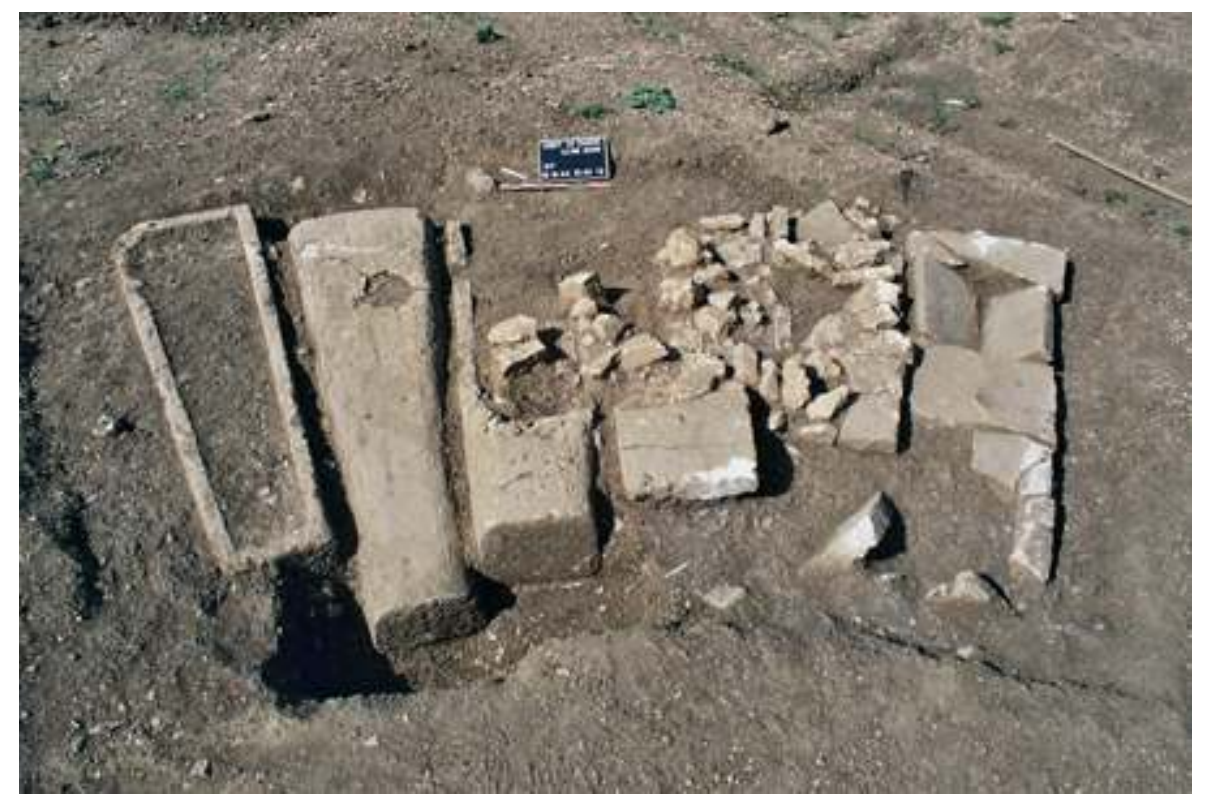

Ligny-le-Châtel, La Coupe au Meunier (cliché P. Chopelain, INRAP)

\section{NOTES DE FIN}

1. Cette proportion rapproche la nécropole de Ligny-le-Châtel de site comme Bierryles-Belles-Fontaines (Yonne) où les sarcophages représentent $35 \%$ des inhumations fouillées, alors qu'à Escolives-Sainte-Camille (site plus proche et sans doute contemporain) les sarcophages représentent à peine plus de $10 \%$ des inhumations fouillées. Pour Escolives et Ligny, les surfaces fouillées sont à peu près identiques alors qu'à Bierry la surface est moindre mais l'occupation funéraire commence dès la fin du $\mathrm{v}^{\mathrm{e}}$ siècle.

2. Abbé CORNAT, Histoire de la ville de Ligny-le-Châtel, département de l'Yonne, Ligny-leChâtel, rééd. YBIS, 1999, 216 p.

3. Un seul exemplaire appartient au Type lithologique 2, mais il s'agit d'un sarcophage $\mathrm{du}$ Groupe $\mathrm{K}$ (cuve et couvercle bipartites taillés dans des blocs de remploi), renvoyant à un tout autre système de production et de diffusion.

4. Les parois et les panneaux de la cuve présentent un décor de lignes pointillées obliques alternées, sur deux registres horizontaux séparés d'une ligne gravée pour les parois, et trois registres verticaux également séparés d'une ligne gravée pour le panneau de tête. Par ce décor, on se rapproche de l'esprit des sarcophages du Groupe A (décor de stries gravées obliques alternées). On remarquera en outre que la préparation du plan de taille, réalisée à la polka, a été conservée, devenant ainsi décorative. 
INDEX

Index géographique : France/Ligny-le-Châtel

Mots-clés : sarcophage 